\title{
Ceftolozane-Tazobactam Treatment of Hypervirulent Multidrug Resistant Pseudomonas aeruginosa Infections in Neutropenic Patients
}

\author{
Paolo E. Coppola ${ }^{1}$, Paolo Gaibani ${ }^{2}$, Chiara Sartor ${ }^{1}$, Simone Ambretti ${ }^{2}$, Russell E. Lewis ${ }^{3,4}$, \\ Claudia Sassi ${ }^{5,6}$, Marco Pignatti ${ }^{6,7}$, Stefania Paolini ${ }^{1}$, Antonio Curti ${ }^{1}$, Fausto Castagnetti ${ }^{1,6}$, \\ Margherita Ursi ${ }^{1}$, Michele Cavo ${ }^{1,6}$ and Marta Stanzani ${ }^{1, *}$ \\ 1 Institute of Hematology "Seràgnoli", IRCCS-Azienda Ospedaliero Policlinico Sant'Orsola-Universitaria di \\ Bologna, 40138 Bologna, Italy; paoloelia.coppola@studio.unibo.it (P.E.C.); chiara.sartor2@unibo.it (C.S.); \\ stefania.paolini@aosp.bo.it (S.P.); antonio.curti2@unibo.it (A.C.); fausto.castagnetti@gmail.com (F.C.); \\ margherita.ursi@studio.unibo.it (M.U.); michele.cavo@unibo.it (M.C.) \\ 2 Microbiology, IRCCS-Azienda Ospedaliero Policlinico Sant'Orsola-Universitaria di Bologna, 40138 Bologna, \\ Italy; paolo.gaibani@unibo.it (P.G.); simone.ambretti@aosp.bo.it (S.A.) \\ 3 Infectious Diseases, IRCCS-Azienda Ospedaliero Policlinico Sant'Orsola-Universitaria di Bologna, \\ 40138 Bologna, Italy; russeledward.lewis@unibo.it \\ 4 Department of Medical and Surgical Sciences (DIMEC)- Università di Bologna, Alma Mater Studiorum, \\ 40138 Bologna, Italy \\ 5 Radiology, IRCCS-Azienda Ospedaliero Policlinico Sant'Orsola-Universitaria di Bologna, 40138 Bologna, \\ Italy; claudia.sassi3@unibo.it \\ 6 Department of Diagnostic and Experimental Medicine Specialty (DIMES)- Università di Bologna, \\ Alma Mater Studiorum, 40138 Bologna, Italy; marco.pignatti@unibo.it \\ 7 Plastic Surgery, IRCCS-Azienda Ospedaliero Policlinico Sant'Orsola-Universitaria di Bologna, \\ 40138 Bologna, Italy \\ * Correspondence: marta.stanzani2@unibo.it
}

Received: 12 November 2020; Accepted: 11 December 2020; Published: 21 December 2020

\begin{abstract}
The effectiveness of ceftolozane/tazobactam for the treatment of infections in neutropenic patients caused by hypervirulent multidrug-resistant (MDR) Pseudomonas aeruginosa has not been previously reported. We identified seven cases of MDR P. aeruginosa infection in neutropenic patients over a four-month period within the same hematology ward. Four cases were associated with rapid progression despite piperacillin-tazobactam or meropenem therapy, and three patients developed sepsis or extensive skin/soft tissue necrosis. In three of the four cases, patients were empirically switched from meropenem to ceftolozane/avibactam before carbapenem susceptibility test results were available, and all four patients underwent extensive surgical debridement or amputation of affected tissues and survived. Further investigation revealed a common bathroom source of MDR P. aeruginosa clonal subtypes ST175 and ST235 that harbored genes for type III secretion system expression and elaboration of ExoU or ExoS exotoxin. We conclude that ceftolozane/tazobactam plus early source control was critical for control of rapidly progressing skin and soft infection in these neutropenic patients caused by highly virulent ST175 and ST235 clones of MDR P. aeruginosa.
\end{abstract}

Keywords: Pseudomonas aeruginosa; ceftolozane/tazobactam; hypervirulent; skin and soft tissue; neutropenia; hematological malignancy

\section{Introduction}

In neutropenic patients, breakthrough infections with Pseudomonas aeruginosa can present as rapidly progressing pneumonia, bacteremia, and sepsis associated with mortality rates of $60-80 \%$ [1]. 
The involvement of other internal organs, limbs, and genitalia is less common but often requires timely surgical intervention in additional to broad-spectrum antibiotics [2,3]. Although the infection often arises endogenously following mucotoxic chemotherapy during neutropenia, exogenous acquisition and horizontal transmission of multidrug-resistant (MDR) strains have been documented via contaminated sinks, toilets, and bathrooms [4-6].

Carbapenems are frequently used as empiric therapy for febrile neutropenia in centers with a high prevalence of extended-spectrum beta-lactamase Enterobacterales, creating pressure for the selection and dissemination of carbapenem-resistant strains [7]. Comparative genomic analysis of MDR $P$. aeruginosa isolates from patients with bloodstream infection has identified dominant clonal sub-types of carbapenem-resistant $P$. aeruginosa (e.g., ST175, ST235) that express virulence attributes such as the type III secretion system (TTSS) [8-10]. This secretion system injects exotoxins, including ExoS, ExoT, ExoU, or ExoY phospholipases, into eukaryotic cells, resulting in distinct patterns of host tissue injury depending on the high-risk clone and site of infection, with ExoU exotoxin associated with the greatest impact on bacterial virulence in human hosts [11]. Therefore, the selection of high-risk MDR and carbapenem-resistant $P$. aeruginosa strains may favor some-high risk clonal lineage with enhanced virulence [10].

Ceftolozane/tazobactam is a novel cephalosporin with activity against gram-negative bacteria, including P. aeruginosa and Escherichia coli [12-14], and has a safety profile similar to other cephalosporins. Ceftolozane/tazobactam is less susceptible to bacterial cell efflux and degradation by multiple classes of beta-lactamases, with reported susceptibility rates for P. aeruginosa of $90 \%$ (including carbapenem-resistant strains) $[12,14,15]$. Therefore, ceftolozane/tazobactam is an appealing monotherapy treatment option compared to older antibiotics that are often used in combination with more toxic aminoglycosides or polymyxins. Although more clinical data in the hematology population is needed, ceftolozane/tazobactam appears to be highly active in the treatment of complicated intra-abdominal and urinary tract infections $[16,17]$, pneumonia [18], and difficult-to-treat (DTR) $P$. aeruginosa [19]. In a recent small single-center case-control study, ceftolozane/tazobactam was well tolerated and at least as effective as other alternatives for $P$. aeruginosa infection in patients with hematologic malignancies, including neutropenic patients [20]. However, it is unknown whether early ceftolozane/tazobactam treatment can improve outcomes of neutropenic patients infected with highly virulent MDR clones of $P$. aeruginosa.

Herein, we report a monocentric outbreak of four cases of hypervirulent MDR P. aeruginosa infection associated with a common identified bathroom source that occurred in the same hematology unit in Italy over a four-month period. All patients, except one, shared the same bathroom, and three patients were treated with ceftolozane/tazobactam for rapidly progressing clinical infection.

\section{Case Descriptions}

Collection of data related to this report was approved by the institutional ethics committee as part of routine infection control surveillance in accordance with the ethical standards laid down in the 1964 Declaration of Helsinki and its later amendments and Italian law. All patients described in this report provided informed consent to present their case history and medical images.

\subsection{Case \#1-Index Case}

A 43-year-old man with acute lymphoblastic leukemia (ALL) in complete remission (CR) was admitted in early February to room C (Figure 1) to receive a fourth cycle of consolidation chemotherapy. Ten days after the end of chemotherapy, while neutropenic, he developed fever with severe sepsis, and increased procalcitonin (PCT, $40 \mathrm{ng} / \mathrm{mL}$ ) and C-reactive protein (CRP, $12 \mathrm{mg} / \mathrm{dL}$ ). Blood cultures were positive for P. aeruginosa that was susceptible to piperacillin/tazobactam, amikacin, cefepime and meropenem. The patient was isolated in a single room with a personal bathroom. 


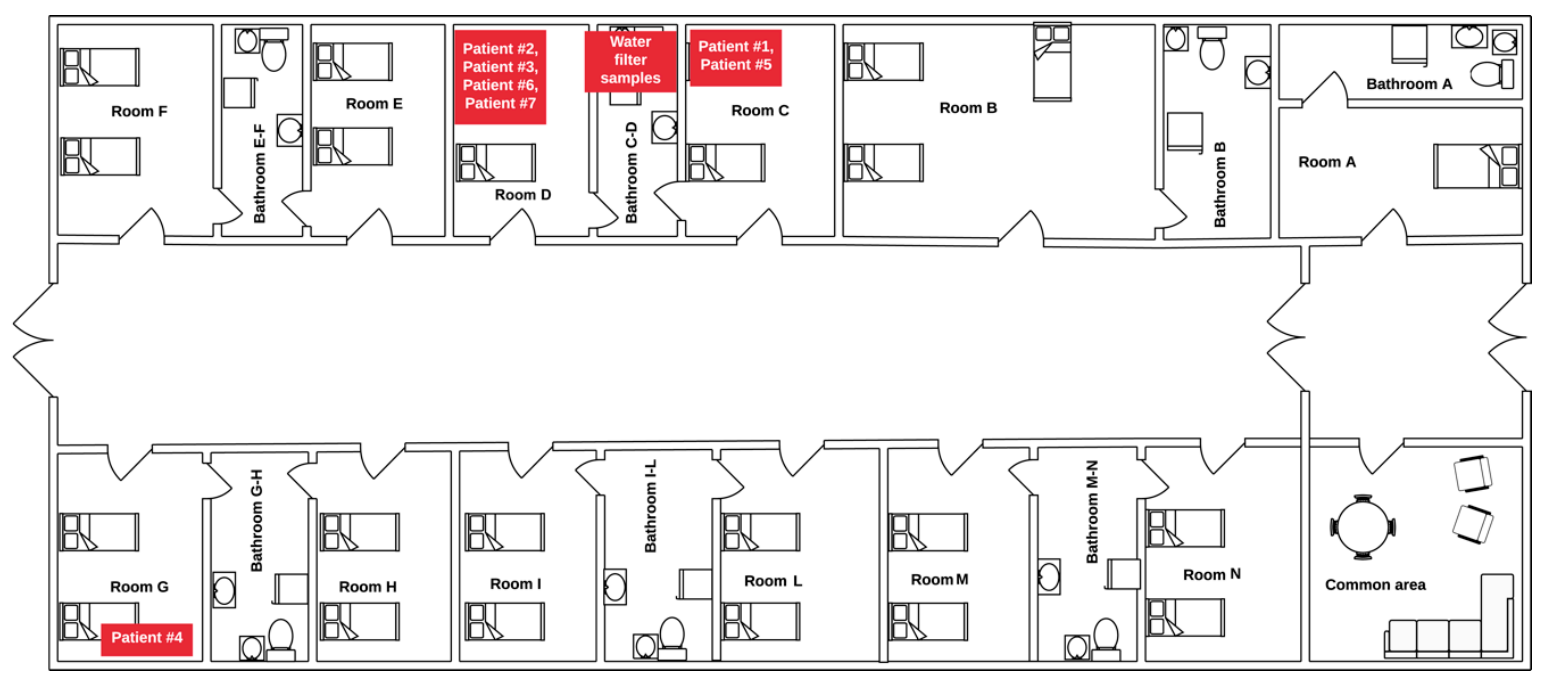

Figure 1. Floorplan of the hematology unit. Six of the reported cases clustered around rooms D and $\mathrm{C}$, which share a bathroom where environmental isolates were collected. One case was detected in a patient from room $\mathrm{G}$.

Unfortunately, the patient remained persistently febrile despite receiving $18 \mathrm{~g}$ of piperacillin/tazobactam by continuous infusion for $>48 \mathrm{~h}$. After switching the patient to $2 \mathrm{~g}$ of meropenem every $8 \mathrm{~h}$ administered in 6-h infusions plus amikacin $15 \mathrm{mg} / \mathrm{kg} /$ day, he improved rapidly and was discharged home without complications.

\subsection{Case \#2}

A 45-year-old man affected by newly diagnosed high-risk acute myeloid leukemia (AML) was admitted January 28 to room D (Figure 1) for induction chemotherapy with fludarabine, cytarabine, and idarubicin. Seven days after the end of the chemotherapy, he presented fever with severe sepsis and began empiric antibiotic therapy with piperacillin/tazobactam. At the fever onset, he reported pain localized under his right toe where a lenticular lesion was visible. We suspected the patient had been washing his feet in the bathroom bidet. After $48 \mathrm{~h}$, blood cultures (BCs) were positive for P. aeruginosa while the skin lesion had now rapidly evolved into blisters (Figure 2A) and the patient developed excruciating pain at palpation of the toe, highly suspicious for necrotizing fasciitis.

Antibiotic susceptibility testing revealed that an isolate was sensitive only to amikacin, colistin, and ceftolozane/tazobactam. His antibiotics were changed to $1.5 \mathrm{~g}$ of ceftolozane/tazobactam every $8 \mathrm{~h}$, 9 million international units (MIU) colistin daily in two divided doses daily following a 9 MIU loading dose, and $15 \mathrm{mg} / \mathrm{kg} /$ day of amikacin.

A computed tomography (CT) scan of the right leg was performed and showed no signs of necrotizing fasciitis (Figure 2C). The right foot blisters were drained, but no purulent colliquated material was recovered (Figure 2B). Unfortunately, the fasciitis worsened over the ensuing $48 \mathrm{~h}$ on antibiotic therapy, and a follow-up CT scan taken 5 days from previous imaging showed infection progressing to the subcutaneous tissue and muscles up to the right lower limb with associated lymphangitis. An amputation of the right leg was deemed necessary. A few days after the operation, the patient improved with resolution of pain and the lymphangitis with progressively reduced erythema. The patient received 16 days of additional antibiotic therapy and recovered from neutropenia 7 days after infection onset, with the aid of granulocyte colony stimulating factor. The patient was discharged home in complete remission of his underlying malignancy without antibiotic therapy. 


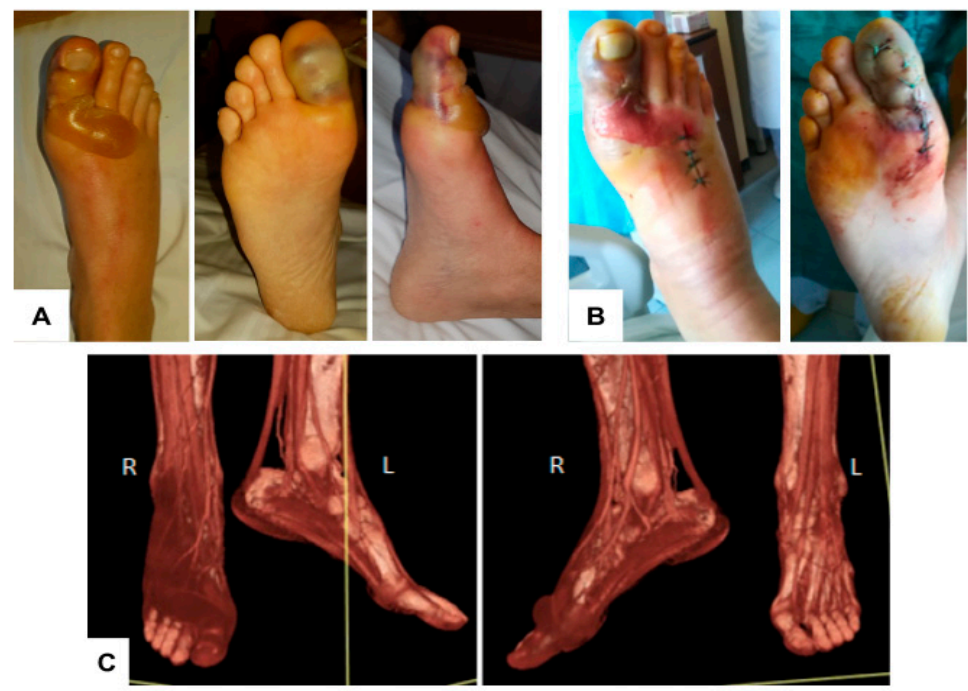

Figure 2. Clinical presentation of $P$. aeruginosa necrotizing fasciitis. (A) The small skin lesion rapidly evolved into blisters; (B) Appearance of the foot after open drainage attempt; (C) Computed tomography (CT) examination of the legs with volume rendering post-processing reformatted images showing the presence of pathologic tissue encasing the vessels, tendons, and muscles of the dorsal region of the right foot and ankle. Only the first toe is involved, while the others are spared.

\subsection{Case \#3}

A 60-year-old man, affected by newly diagnosed AML, was admitted on February 19 to room $C$ (Figure 1). The patient received induction chemotherapy, to which he was refractory, followed by a cycle of rescue chemotherapy. After 12 days from the end of the second cycle, he developed fever with severe sepsis complicated by initial balanoposthitis. Empiric antibiotic therapy was started with meropenem, but the lesion evolved rapidly with painful erythema and edema of pubis, penis, scrotum, perineal, and inguinal lymphadenopathy.

Superficial necrosis similar to Fournier's gangrene (Figure 3A-C) was present on the penis. An ultrasound revealed soft tissue imbibition, signs of epididymitis, and conserved parenchyma of penis and testicles. A chest and abdominal CT scan were also performed that did not identify other infection localization. After 2 days from the fever onset, the BCs turned positive for an MDR P. aeruginosa sensitive to meropenem, imipenem, and ceftolozane/tazobactam.

Considering the rapid progression of the infection and previous cases, we immediately started antibiotic therapy with ceftolozane/tazobactam, colistin, amikacin, meropenem, and clindamycin, with the latter two antibiotics later discontinued within $48 \mathrm{~h}$. The patient improved rapidly with reduced fever and stabilization of erythema and necrosis (Figure 3D). To accelerate the healing of necrotic tissue, the urologist initiated hyperbaric therapy sessions. After eight 1-h hyperbaric therapy sessions and continued antibiotic therapy (ceftolozane/tazobactam and meropenem), edema, lymphadenopathy, and erythema resolved. A dry necrotic dorsal eschar persisted (Figure 3E) that was treated by plastic surgeons with debridement (Figure 3F), full thickness skin graft (Figure 3G), and negative pressure dressing (Figure $3 \mathrm{H}$ ) for 5 days to improve skin graft take (Figure 3I). The patient achieved a CR for his hematological malignancy and was discharged after 16 days from the beginning of the infectious episode. 

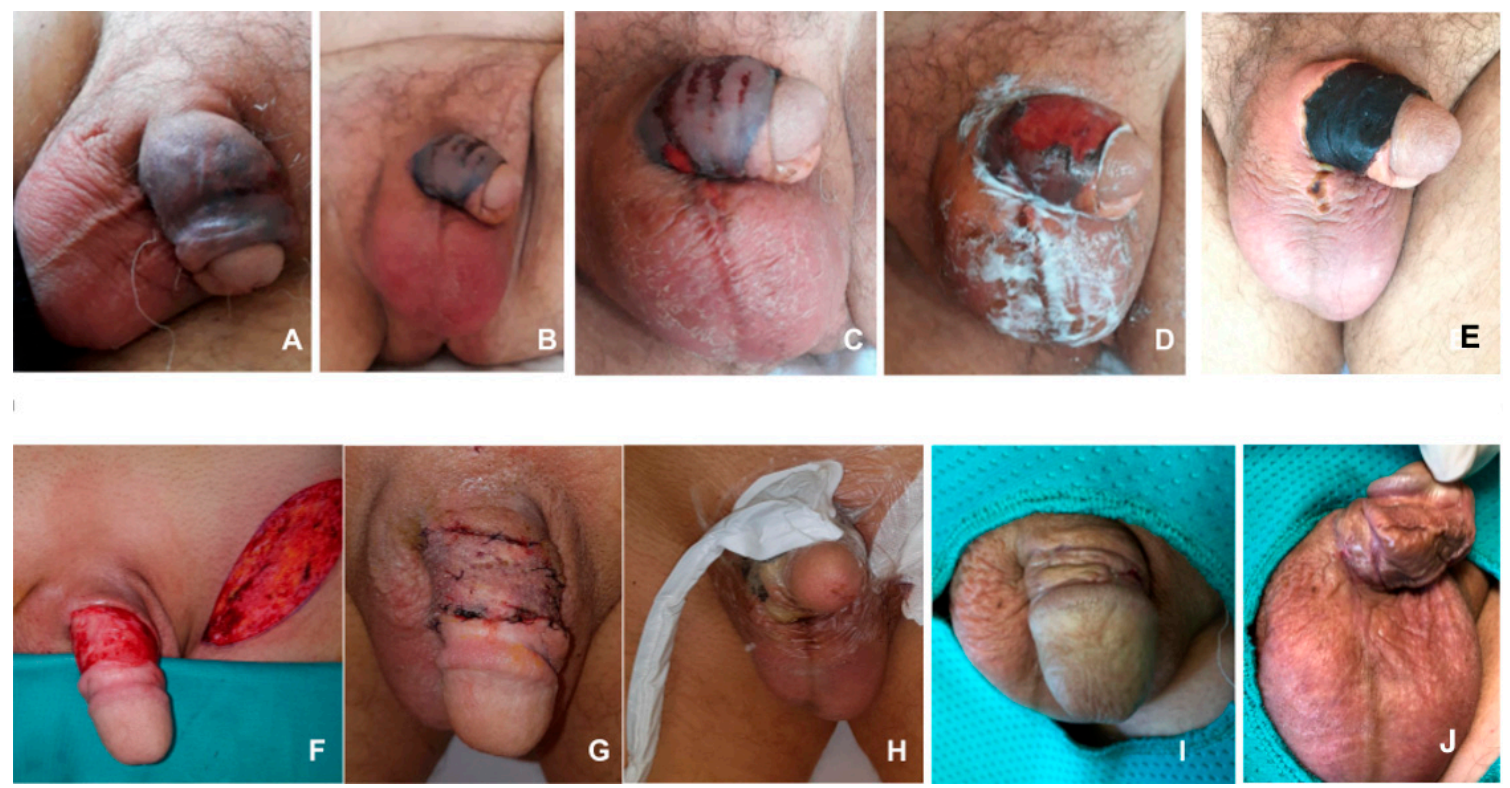

Figure 3. Clinical presentation of P. aeruginosa Fournier's gangrene. (A-D) Evolving infection of penis and scrotum; (E) Development of a dry necrotic dorsal eschar; (F) Debridement of necrotic tissue of the penis shaft and full thickness skin taken from left inguinal area; (G) Full thickness skin grafted on the soft tissue loss; (H) Negative pressure dressing positioned over the skin graft to improve skin graft take (I,J).

\subsection{Case \#4}

A 64-year-old woman affected by B-follicular lymphoma was admitted on April 4 in room G (Figure 1) to undergo autologous stem-cell transplantation. The transplant was performed on April 24 , and the following phase of neutropenia was complicated 10 days later by an episode of septic shock with hepatic failure, serum hyperosmolarity, hypotension, and atrial fibrillation. P. aeruginosa was detected within $24 \mathrm{~h}$ of blood culture. Empiric antibiotic therapy was promptly changed from meropenem plus amikacin to ceftolozane-tazobactam. The patient developed multiorgan failure (MOF) and was transferred to the ICU where she was clinically improved.

After $48 \mathrm{~h}$, she was transferred back to the hematology unit to continue ceftolozane/ tazobactam and clinical support. The patient continued ceftolozane/tazobactam for 14 days and then was discharged home after 19 days in complete hematologic remission.

\subsection{Cases \#5-7}

Three more patients hospitalized in the rooms $C$ and $\mathrm{D}$ developed less severe infections by $P$. aeruginosa during the same time. Case 5 was a 63-year-old male affected by multiple myeloma who presented an asymptomatic urinary infection by $P$. aeruginosa sensitive to meropenem and amikacin at the beginning of March, which did not require treatment. Case 6 was a 67 -year-old man with mantle B-cell lymphoma who presented with P. aeruginosa sensitive only to colistin and ceftolozane-tazobactam that resolved quickly with ceftolozane-tazobactam and neutrophil recovery. Patient 7, a 72-year-old man affected by AML, who was hospitalized in room D during February and March, died at an outside hospital in May for a severe sepsis caused by P. aeruginosa. He did not receive treatment with ceftolozane/tazobactam.

Because nearly all the P. aeruginosa infections involved patients admitted to rooms C and D (Table 1), we performed environmental microbiological surveys in the shared bathroom between the two involved rooms, checking for 20 sites of possible common source in the shared bathrooms, rooms, and common areas. P. aeruginosa was isolated in the water trap filter and in the tap water of the 
bathroom bidet. After the filter removal and cleaning, no other P. aeruginosa infections were detected in this unit after 8 months of follow-up.

Table 1. Clinical characteristics and outcomes of patients with P. aeruginosa infection.

\begin{tabular}{|c|c|c|c|c|}
\hline ID & Case 1 & Case 2 & Case 3 & Case 4 \\
\hline Room & C & $\mathrm{D}$ & $\mathrm{D}$ & G \\
\hline $\begin{array}{l}\text { Isolate } \\
\text { (MLST type) }\end{array}$ & N/A & N/A & $\begin{array}{l}\text { PABO62 } \\
\text { (ST235) }\end{array}$ & $\begin{array}{l}\text { PABO48 } \\
\text { (ST175) }\end{array}$ \\
\hline Date of onset & Feb 8 & Feb 19 & April 7 & May 4 \\
\hline $\begin{array}{l}\text { Hematological } \\
\text { disease }\end{array}$ & ALL & AML & AML & $\mathrm{LY}$ \\
\hline Age & 43 & 44 & 60 & 64 \\
\hline Disease status & CR & Onset & Onset & Relapse \\
\hline Chemotherapy & Consol. & Induction & Induction & Relapse \\
\hline Neutropenia & Yes & Yes & Yes & Yes \\
\hline $\begin{array}{l}\text { Antimicrobial } \\
\text { prophylaxis }\end{array}$ & TMP-SMX & TMP-SMX, levofloxacin & TMP-SMX & $\begin{array}{l}\text { TMP-SMX, } \\
\text { levofloxacin }\end{array}$ \\
\hline Site of infection & Blood & Blood, skin, muscle & Blood, genitals & Blood, CVC \\
\hline Culture site & Peripheral blood & Peripheral blood & Peripheral blood & CVC \\
\hline $\begin{array}{l}\text { Clinical } \\
\text { presentation }\end{array}$ & Severe sepsis & Necrotizing fasciitis & Fournier's gangrene & Septic shock \\
\hline Treatment & MER & $\begin{array}{l}\mathrm{C} / \mathrm{T}+\mathrm{COL}+\mathrm{AMK}+\mathrm{MER} \\
\text { surgery }\end{array}$ & $\begin{array}{l}\mathrm{C} / \mathrm{T}+\mathrm{COL}+\mathrm{AMK}+\mathrm{CLI} ; \\
\text { Surgery }\end{array}$ & $\mathrm{C} / \mathrm{T}$ \\
\hline Outcome & Alive & Alive (leg amputation) & Alive (plastic surgery) & Alive \\
\hline
\end{tabular}

ALL: acute lymphoblastic leukemia; AML; acute myeloblastic leukemia; CR: complete remission; TMP-SMX: trimethoprim-sulfamethoxazole; AMK: amikacin; CEF: cefepime; PTZ: piperacillin-tazobactam; MER: meropenem; COL: colistin; C/T: ceftolozane-tazobactam; IMP: imipenem; CLI: clindamycin, ASCT: autologous stem-cell transplantation; CVC: central venous catheter; N/A: Not Applicable.

\section{Microbiology}

P. aeruginosa strains were identified by MALDI-TOF MS assay (Bruker Daltonics, Leipzig, Germany). Antimicrobial susceptibility testing was performed by Microscan (Beckman Coulter, Brea USA) and confirmed by MIC test strip (Liofilchem, Roseto degli Abruzzi, Italy). MIC results were interpreted following EUCAST clinical breakpoints v9.0. Previous cumulative susceptibility reports from our institution indicated that $92 \%$ of all tested $P$. aeruginosa isolates ( $96 \%$ from blood samples) were susceptible to ceftolozane/tazobactam. Whole-genome sequencing was performed as previously described [9]. Briefly, libraries were prepared by the Nextera XT sample preparation kit and sequenced using the Illumina MiSeq platform (Illumina, San Diego, USA) with a $2 \times 250$ paired-end run. All read sets were evaluated by FastQC software and then assembled with SPAdes v.3.10 with careful settings.

Unfortunately, genomes from clinical strains isolated from patients 1 and 2 were not included in the analysis because isolates were not available at the time of sequencing. Assembled genomes were screened for known antimicrobial resistance, sequence type (ST), and phage regions by CGE server (https://cge.cbs.dtu.dk/services/MLST/), Pubmlst(https://pubmlst.org/bigsdb?db=pubmlst_ paeruginosa_seqdef) and PHAGE (http://phast.wishartlab.com) web tools. A core genome single nucleotide polymorphism (SNP) phylogeny was generated by Parsnp using the complete genome of strain PAO1 as reference. The phylogenetic tree was edited and visualized using FigTree software v1.4.3 (http://tree.bio.ed.ac.uk/software/figtree/). The whole-genome project is available at EMBL/ENA/EBI under study number PRJEB34087.

A summary of phenotypic and genotypic traits of $P$. aeruginosa strains analyzed in this study are shown in Tables 2 and 3. Antimicrobial susceptibility profiles of clinical $P$. aeruginosa strains demonstrated patient isolates from cases 3 and 4 were susceptible to ceftolozane/tazobactam, ceftazidime/avibactam, and colistin, but resistant to gentamicin, ceftazidime, and piperacillin/tazobactam. In addition, 4/5 P. aeruginosa clinical isolates were resistant to imipenem and cefepime. 
Table 2. Phenotypic characteristics of Pseudomonas aeruginosa strains isolated from patients and the environment.

\begin{tabular}{|c|c|c|c|c|c|c|c|c|c|c|c|}
\hline \multirow[b]{2}{*}{ Strain (Source) } & \multirow[b]{2}{*}{ Sample } & \multirow[b]{2}{*}{ CAZ } & \multirow[b]{2}{*}{ FEP } & \multirow[b]{2}{*}{ IPM } & \multirow[b]{2}{*}{ MEM } & \multicolumn{3}{|c|}{$\operatorname{MIC}(\mu \mathrm{g} / \mathrm{mL})$} & \multirow[b]{2}{*}{ CST } & \multirow[b]{2}{*}{ GEN } & \multirow[b]{2}{*}{ AMK } \\
\hline & & & & & & TZP & CAZ/AVI & $\mathrm{C} / \mathrm{T}$ & & & \\
\hline B062 (Case \#3) & Blood & 32 & $>8$ & $<1$ & 1 & $>16$ & 4 & $<1$ & 0.5 & $>4$ & 16 \\
\hline BO48 (Case \#4) & Blood & 16 & $>8$ & $>8$ & 16 & $>16$ & 4 & $<1$ & 0.5 & $>4$ & 16 \\
\hline BO68 (Case \#6) & Blood & 32 & $>8$ & $>8$ & 8 & $>16$ & 4 & $<1$ & 0.5 & $>4$ & $<8$ \\
\hline BO93 (bidet) & $\begin{array}{l}\text { Bidet } \\
\text { filter }\end{array}$ & 32 & $>8$ & 8 & 16 & $>16$ & $>8$ & $>16$ & 0.5 & $>4$ & $>16$ \\
\hline BO94 (bidet) & $\begin{array}{l}\text { Bidet } \\
\text { water }\end{array}$ & 32 & $>8$ & $>8$ & 16 & $>16$ & $>8$ & $>16$ & 0.5 & $>4$ & $>16$ \\
\hline
\end{tabular}

Caz, ceftazidime; FEP, cefepime; IPM, imipenem; MEM, meropenem; TZP, piperacillin-tazobactam; CAZ/AVI, ceftazidime/avibactam; C/T, ceftolozane/tazobactam; CST, colistin; GEN, gentamicin; AMK, amikacin.

Table 3. Genotypic characteristics of Pseudomonas aeruginosa strains isolated from patients and the environment.

\begin{tabular}{|c|c|c|c|c|c|c|c|}
\hline Strain (Source) & ST & Carbapenemase & Beta-Lactams & Amino-Glycosides & Fluoro-Quinolones & Sulfon-Amide & $\begin{array}{c}\text { No. } \\
\text { PhageRegions }\end{array}$ \\
\hline B062 (Case \#3) & 235 & & $\begin{array}{c}\text { blaOXA- } 488 \\
\text { blaPAO }\end{array}$ & $\begin{array}{c}\text { aac }\left(6^{\prime}\right)-\mathrm{Ib} 3, \text { aadA11, } \\
\text { ant }\left(2^{\prime \prime}\right)-\mathrm{Ia}, \\
\text { aph }\left(3^{\prime}\right)-\mathrm{Il} b\end{array}$ & $a a c\left(6^{\prime}\right)-I b-c r$ & sul1 & 9 \\
\hline BO48 (Case \#4) & 175 & & $\begin{array}{l}\text { blaOXA-50, } \\
\text { blaPAO, }\end{array}$ & $\begin{array}{l}\text { ant }\left(2^{\prime \prime}\right)-\mathrm{Ia}, \\
\operatorname{aph}\left(3^{\prime}\right)-\mathrm{Il} b\end{array}$ & $\operatorname{crpP}$ & sul1 & 11 \\
\hline BO68 (Case \#6) & 175 & & $\begin{array}{l}\text { blaOXA-50, } \\
\text { blaPAO, }\end{array}$ & $\begin{array}{l}\operatorname{ant}\left(2^{\prime \prime}\right)-I a, \\
\operatorname{aph}\left(3^{\prime}\right)-I i b\end{array}$ & $\operatorname{crpP}$ & sul1 & 11 \\
\hline BO93 (bidet) & 235 & blaVIM-1 & $\begin{array}{c}\text { blaOXA-488, } \\
\text { blaPAO }\end{array}$ & 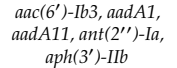 & $a a c\left(6^{\prime}\right)-I b-c r$ & sul1 & 12 \\
\hline BO94 (bidet) & 235 & blaVIM-1 & $\begin{array}{l}\text { blaOXA-488, } \\
\text { blaPAO }\end{array}$ & $\begin{array}{l}\text { Ib3, aadA1, aad } A 11, \\
\text { ant }\left(2^{\prime \prime}\right)-I a \\
\text { aph }\left(3^{\prime}\right)-I b\end{array}$ & $a a c\left(6^{\prime}\right)-I b-c r$ & sul1 & 12 \\
\hline
\end{tabular}

ST, sequence type.

At the same, time, environmental P. aeruginosa strains isolated from the bidets were resistant against all antimicrobials except colistin. Genetic analysis showed that PABO48 and PABO68 (strains isolated from cases 4 and 6) harbored the same genes coding for antimicrobial resistance to $\beta$-lactams, aminoglycoside, fluoroquinolone, and sulfonamide and possessed similar prophage regions. Similarly, PABO62 (strain isolated from patient 3) and PABO93 and PABO94 (strains isolated from the water and filter in bathroom C-D) shared a similar genetic antimicrobial gene pattern. Of note, the two environmental $P$. aeruginosa strains harbored carbapenemase gene blaVIM-1.

MLST analysis demonstrated that PABO48 and PABO68 strains belonged to ST175, while PABO62 and two environmental strains (i.e., PABO93 and PABO94) belonged to the ST235. A phylogenetic tree constructed based on the core genome SNPs analysis of $P$. aeruginosa genomes revealed that clinical isolates PABO68 and PABO48 clustered within the ST175 group, whereas clinical strain PABO62 and environmental isolates PABO93 and PABO94 grouped closely within the ST235 group (Figure 4). A deeper examination of the genomic relationship between P. aeruginosa strains included in this study demonstrated that PABO48 and PABO68 isolates clustered closely within the ST175 group and PABO62, PABO93, and PABO94 strains formed a monophyletic group within ST235 (Supplemental Materials Figures S1 and S2). 


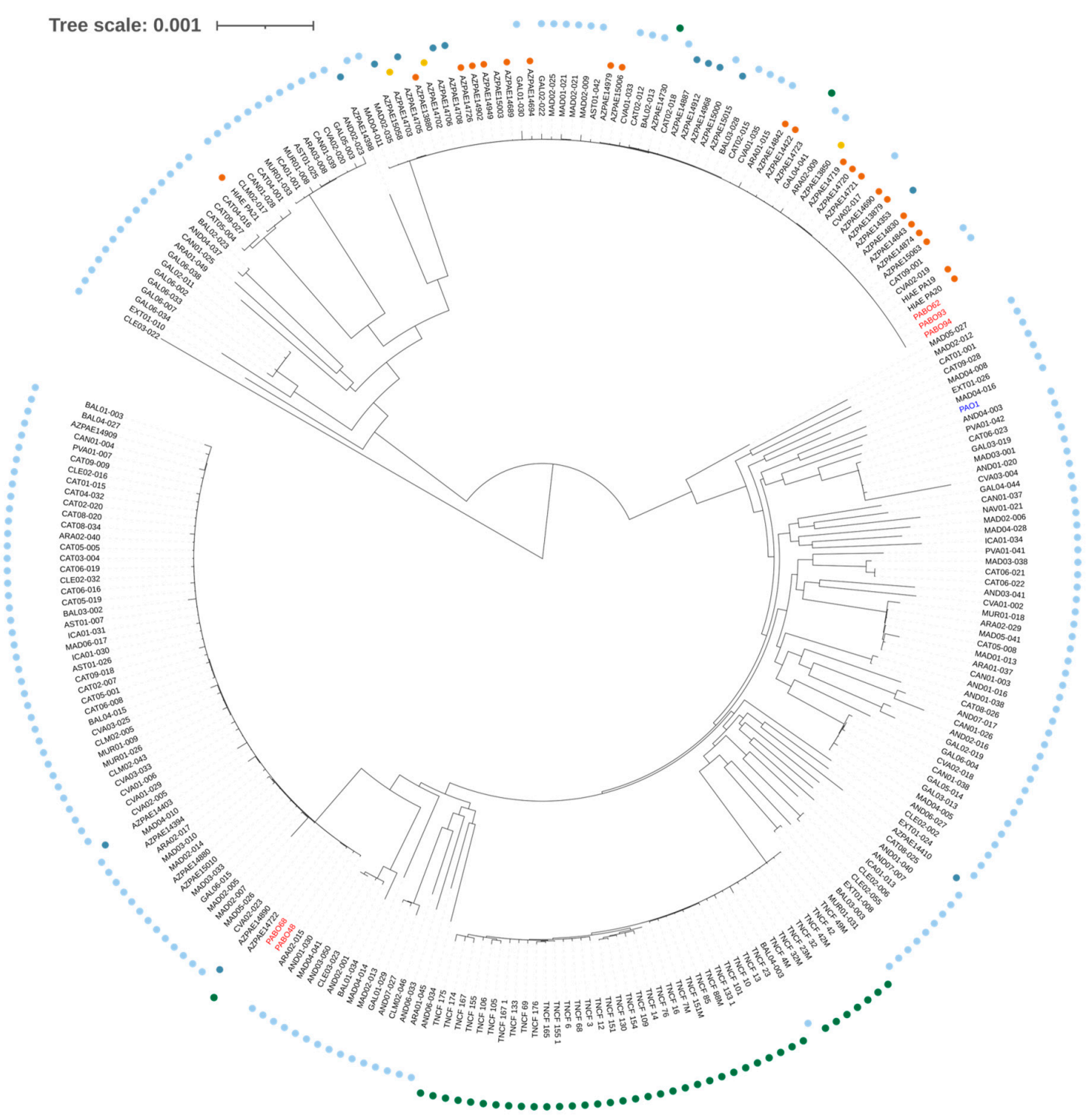

Figure 4. Maximum likelihood phylogeny based on the core genome SNP of the Pseudomonas aeruginosa genomes. The P. aeruginosa strains (clinical and environmental isolates) included in this study are written in red font, and the references strain is written in blue font. Yellow circles indicate strains isolated in Asia, orange circles indicate strains isolated from North and South America, light blue circles indicate strains isolated from Spain, green circles indicate strains isolated from Italy, and dark blue circles indicate strains isolated from other European Union countries.

Finally, we identified genes in both patient and environmental isolates reported to influence the virulence of $P$. aeruginosa infections. These included genes involved in (i) alginate production (alg and $m u c$ ); (ii) flagella (fle, flg, flg), lipopolysaccharide (waa), and type IV pili expression (chp, fim, pil, and $x c p A / p i l D)$; (iii) rhamnolipid production ( $r h l A$ and $r h l B)$, iron uptake through pyochelin (fpt, pch), and pyoverdine $(f p v A, p r d)$ siderophores; (iv) pyocyanin pigment production $(p h n A / B),(v)$ aprA, lasA, lasB protease secretion; (vi) quorum molecule responsive transcriptional regulators (Las1, LasR, Rhll, rh1R), (vii) Hcp secretion island-1 encoded type VI secretion system (iclp, ppk, icm, dot, his, hcp, lipd, vrg, fha, tag); (viii) type-III secretion systems ( $p s c, p c r$, exs, pop); and (ix) Xcp extracellular protein secretion system (exp gene family). All sequences isolates expressed the capacity for producing $\mathrm{plcH}$ and ToxA exotoxin. However, ST175 isolates BO48 and BO68 also harbored exoT, exoU and exoY and exoS, exoT, and exoY, respectively. Among strains belonging to clonal subtype ST235, the patient isolate BO62 also 
harbored exoS, ExoT, and exoY, while the isolates recovered from the bidet water (BO93) and filter (BO94) also contained exoT, exoU, and exoY and exoS, exoT, exoU, and exoY, respectively.

\section{Discussion}

This limited experience suggests that ceftolozane/tazobactam may be an effective treatment option for hypervirulent MDR P. aeruginosa infections in neutropenic patients that were likely acquired from a common bathroom source. Aggressive source control requiring amputation (Case 2) and hyperbaric therapy to enhance tissue healing and accelerated regeneration (Case 3) were used in combination with ceftolozane/tazobactam-based regimens.

Clinical data concerning the efficacy of ceftolozane/tazobactam for the treatment of severe $P$. aeruginosa infections in patients with hematological malignancies is limited. In a small case-control study, Fernández-Cruz et al. compared the outcomes of 19 neutropenic patients with P. aeruginosa who received ceftolozane/tazobactam monotherapy versus 38 controls who received other antibiotics [20]. The authors found no significant differences in clinical cure at day $14(89.5 \%$ versus $71.1 \% ; p=0.183)$ or recurrence $(15.8 \%$ versus $10.5 \% ; p=0.675)$. However, 30-day mortality was lower among ceftolozane/tazobactam-treated patients $(5.3 \%$ versus $28.9 \% ; p=0.045)$. The authors concluded that ceftolozane-tazobactam was well tolerated and as effective as other alternatives for $P$. aeruginosa infection in patients with hematologic malignancy, including neutropenic patients with sepsis caused by extreme-drug resistant (XDR) strains.

We believe the early switch to ceftolozane/tazobactam at the first clinical suspicion of MDR and early source control were critical factors in the survival of our patients. Among the patient and environmental isolates available for analysis, sequencing confirmed that the P. aeruginosa isolates recovered from patients and environmental sources belonged to high-risk ST175 and ST235 clones, which have been linked to the global emergence of MDR strains resistant to fluoroquinolones and a capacity for rapid emergence of resistance to $\beta$-lactams and carbapenems through mutation and acquisition of resistance elements [8,9]. These high-risk clones are associated with increased risk of treatment failure due not only to antibiotic resistance, but also their frequent expression of a number of established virulence factors such as type III secretion systems that elaborate potent exotoxins such as the phospholipase ExoU, which is often associated with extensive tissue destruction and localized immunosuppression [11].

In conclusion, the clinical presentation of a high-virulent Pseudomonas infection may be an important clinical sign that an isolate is a high-risk clonal subtype predisposed to rapid emergence of MDR, thus supporting early use of ceftolozane/tazobactam in neutropenic patients until susceptibility is established and clinical infection has stabilized.

Supplementary Materials: The following supplemental figures are available online at http://www.mdpi.com/ 2076-2607/8/12/2055/s1: Figure S1: Maximum likelihood phylogenetic tree based on the SNPs in the core genomes of ST175 Pseudomonas aeruginosa clinical strains; Figure S2: Maximum likelihood phylogenetic tree based on the SNPs in the core genomes of ST235 Pseudomonas aeruginosa environmental strains.

Author Contributions: P.E.C., M.S., and R.E.L. drafted and revised the manuscript; P.G. and S.A. provided microbiology data; S.P., A.C., C.S. (Chiara Sartor), F.C., and M.U. and M.C.; provided clinical information; C.S. (Claudia Sassi) provided radiology images; M.P. provided data on plastic surgery. All authors have read and agreed to the published version of the manuscript.

Funding: This research received no external funding.

Conflicts of Interest: The authors declare no conflict of interest.

\section{References}

1. Smibert, O.; Satlin, M.J.; Nellore, A.; Peleg, A.Y. Carbapenem-Resistant Enterobacteriaceae in Solid Organ Transplantation: Management Principles. Curr. Infect. Dis. Rep. 2019, 21, 26. [CrossRef] [PubMed]

2. Emmett, C.D.; Kane, G. Necrotising fasciitis caused by P aeruginosa in a male patient with chronic lymphocytic leukaemia. BMJ Case Rep. 2013, 2013. [CrossRef] [PubMed] 
3. Martinelli, G.; Alessandrino, E.; Bernasconi, P.; Caldera, D.; Colombo, A.A.; Malcovati, L.; Gaviglio; Vignoli, G.; Borroni, G.; Bernasconi, C. Fournier's gangrene: A clinical presentation of necrotizing fasciitis after bone marrow transplantation. Bone Marrow Transplant. 1998, 22, 1023-1026. [CrossRef]

4. Verweij, P.E.; Bijl, D.; Melchers, W.J.; De Pauw, B.E.; Meis, J.F.; Hoogkamp-Korstanje, J.A.; Voss, A. Pseudo-outbreak of multiresistant Pseudomonas aeruginosa in a hematology unit. Infect. Control Hosp. Epidemiol. 1997, 18, 128-131. [CrossRef] [PubMed]

5. Breathnach, A.; Cubbon, M.; Karunaharan, R.; Pope, C.; Planche, T. Multidrug-resistant Pseudomonas aeruginosa outbreaks in two hospitals: Association with contaminated hospital waste-water systems. J. Hosp. Infect. 2012, 82, 19-24. [CrossRef] [PubMed]

6. Kossow, A.; Kampmeier, S.; Willems, S.; Burckhardt, B.; Groth, C.; Kipp, F.; Mellmann, A.; Stelljes, M.; Berdel, W.E.; Groll, A.; et al. Control of Multidrug-Resistant Pseudomonas aeruginosa in Allogeneic Hematopoietic Stem Cell Transplant Recipients by a Novel Bundle Including Remodeling of Sanitary and Water Supply Systems. Clin. Infect. Dis. 2017, 65, 935-942. [CrossRef] [PubMed]

7. Tamma, P.D.; Rodríguez-Baño, J. The Use of Noncarbapenem $\beta$-Lactams for the Treatment of Extended-Spectrum $\beta$-Lactamase Infections. Clin. Infect. Dis. 2017, 64, 972-980. [CrossRef] [PubMed]

8. Treepong, P.; Kos, V.; Guyeux, C.; Blanc, D.; Bertrand, X.; Valot, B.; Hocquet, D. Global emergence of the widespread Pseudomonas aeruginosa ST235 clone. Clin. Microbiol. Infect. 2018, 24, 258-266. [CrossRef] [PubMed]

9. Cabot, G.; López-Causapé, C.; Ocampo-Sosa, A.A.; Sommer, L.M.; Domínguez, M.Á.; Zamorano, L.; Juan, C.; Tubau, F.; Rodríguez, C.; Moyà, B.; et al. Deciphering the Resistome of the Widespread Pseudomonas aeruginosa Sequence Type 175 International High-Risk Clone through Whole-Genome Sequencing. Antimicrob. Agents Chemother. 2016, 60, 7415-7423. [PubMed]

10. Peña, C.; Cabot, G.; Gómez-Zorrilla, S.; Zamorano, L.; A Ocampososa, A.; Murillas, J.; Almirante, B.; Pomar, V.; Aguilar, M.; Granados, A.; et al. Influence of Virulence Genotype and Resistance Profile in the Mortality of Pseudomonas aeruginosa Bloodstream Infections. Clin. Infect. Dis. 2015, 60, 539-548. [CrossRef]

11. Engel, J.N.; Balachandran, P. Role of Pseudomonas aeruginosa type III effectors in disease. Curr. Opin. Microbiol. 2009, 12, 61-66. [CrossRef]

12. Van Duin, D.; Bonomo, R.A. Ceftazidime/Avibactam and Ceftolozane/Tazobactam: Second-generation $\beta$-Lactam/ $\beta$-Lactamase Inhibitor Combinations. Clin. Infect. Dis. 2016, 63, 234-241. [CrossRef] [PubMed]

13. Zhanel, G.G.; Chung, P.; Adam, H.; Zelenitsky, S.; Denisuik, A.; Schweizer, F.; Lagacé-Wiens, P.R.S.; Rubinstein, E.; Gin, A.S.; Walkty, A.; et al. Ceftolozane/Tazobactam: A Novel Cephalosporin/ß-Lactamase Inhibitor Combination with Activity Against Multidrug-Resistant Gram-Negative Bacilli. Drugs 2013, 74, 31-51. [CrossRef] [PubMed]

14. Cluck, D.; Lewis, P.; Stayer, B.; Spivey, J.; Moorman, J. Ceftolozane-tazobactam: A new-generation cephalosporin. Am. J. Heal. Pharm. 2015, 72, 2135-2146. [CrossRef] [PubMed]

15. Goodlet, K.J.; Nicolau, D.P.; Nailor, M.D. In Vitro Comparison of Ceftolozane-Tazobactam to Traditional Beta-Lactams and Ceftolozane-Tazobactam as an Alternative to Combination Antimicrobial Therapy for Pseudomonas aeruginosa. Antimicrob. Agents Chemother. 2017, 61, e01350-17. [CrossRef] [PubMed]

16. Solomkin, J.; Hershberger, E.; Miller, B.; Popejoy, M.; Friedland, I.; Steenbergen, J.; Yoon, M.; Collins, S.; Yuan, G.; Barie, P.S.; et al. Ceftolozane/Tazobactam Plus Metronidazole for Complicated Intra-abdominal Infections in an Era of Multidrug Resistance: Results From a Randomized, Double-Blind, Phase 3 Trial (ASPECT-cIAI). Clin. Infect. Dis. 2015, 60, 1462-1471. [CrossRef] [PubMed]

17. Scott, L.J. Ceftolozane/Tazobactam: A Review in Complicated Intra-Abdominal and Urinary Tract Infections. Drugs 2016, 76, 231-242. [CrossRef] [PubMed]

18. Haidar, G.; Philips, N.J.; Shields, R.K.; Snyder, D.; Cheng, S.; A Potoski, B.; Doi, Y.; Hao, B.; Press, E.G.; Cooper, V.S.; et al. Ceftolozane-Tazobactam for the Treatment of Multidrug-Resistant Pseudomonas aeruginosa Infections: Clinical Effectiveness and Evolution of Resistance. Clin. Infect. Dis. 2017, 65, 110-120. [CrossRef] [PubMed]

19. Tamma, P.D.; Aitken, S.L.; A Bonomo, R.; Mathers, A.J.; Van Duin, D.; Clancy, C.J. OUP accepted manuscript. Clin. Infect. Dis. 2020. [CrossRef] 
20. Fermández-Cruz, A.; Alba, N.; Semiglia-Chong, M.A.; Padilla, B.; Rodríguez-Macías, G.; Kwon, M.; Cercenado, E.; Chamorro-De-Vega, E.; Machado, M.; Pérez-Lago, L.; et al. A Case-Control Study of Real-Life Experience with Ceftolozane-Tazobactam in Patients with Hematologic Malignancy and Pseudomonas aeruginosa Infection. Antimicrob. Agents Chemother. 2018, 63, e02340-18. [CrossRef]

Publisher's Note: MDPI stays neutral with regard to jurisdictional claims in published maps and institutional affiliations.

(C) 2020 by the authors. Licensee MDPI, Basel, Switzerland. This article is an open access article distributed under the terms and conditions of the Creative Commons Attribution (CC BY) license (http://creativecommons.org/licenses/by/4.0/). 